\title{
L'image des langues minoritaires à travers les médias contemporains
}

A minority language's image in the contemporary media

\section{Ruth Lysaght}

\section{Q OpenEdition}

12 Journals

Édition électronique

URL : https://journals.openedition.org//bl/1898

DOI : $10.4000 / \mathrm{lbl} .1898$

ISSN : 2727-9383

Éditeur

Université de Bretagne Occidentale - UBO

\section{Édition imprimée}

Date de publication : 1 février 2013

Pagination : 233-252

ISBN : 979-10-92331-00-4

ISSN : 1270-2412

\section{Référence électronique}

Ruth Lysaght, "L'image des langues minoritaires à travers les médias contemporains », La Bretagne Linguistique [En ligne], 17 | 2013, mis en ligne le 01 mai 2021, consulté le 22 mai 2021. URL : http:// journals.openedition.org/lbl/1898; DOI : https://doi.org/10.4000/lbl.1898

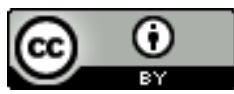

La Bretagne Linguistique est mise à disposition selon les termes de la Licence Creative Commons Attribution 4.0 International. 
Ruth LYSAGHT*

\section{L'image des langues minoritaires à travers les médias contemporains}

\section{$\mathrm{T}$} oute langue fournit un ensemble particulier de métaphores à travers lesquelles ses locuteurs approchent leur environnement (Ó Laoire, 2005). Toute langue a également une image externe : la façon dont elle est perçue par ses locuteurs et par des non-locuteurs. Les médias audiovisuels en général, et la télévision en particulier, influencent ces deux aspects de l'image d'une langue, en la rendant plus visible et en accélérant l'évolution linguistique.

L'ex-directeur des émissions à TG4 ${ }^{1}$, Cilian Fennell, tente de définir ce qui compose une langue, faisant référence à l'irlandais :

Cén dath atá ar an dteanga? Tá sí iontach saibhir... [Braitheann an íomhá ar an gcaoi ina bhfuil an teanga] labharta, scríofa, agus feicthe. [Quelle 'image' a la langue irlandaise ? Elle est très riche... [L'image dépend de la manière dont une langue est prononcée, écrite et la manière dont elle est perçue] (Fennell, 2001).

L'image d'une langue est importante, étant liée à l'image de soi. Pour les locuteurs, leur langue fait partie d'un réseau englobant

\footnotetext{
* Post-doctorante en celtique en 2012, CRBC (EA 4451), UBO/ueb

1. TG4 est la chaîne de télévision nationale de la langue irlandaise (www.tg4.ie).
} 
estime de soi, autonomie, identité et respect (Kāretu, 1993 : 224-6; Mac Aodha Bhuí, 2010). Pour les autres, une langue différente peut susciter la curiosité, la peur, une attitude défensive, l'humilité ou bien peut provoquer une nouvelle perspective (Sinha, 2005; Waters, 2006). Comme le dit Séamus Deane (1985), il est difficile d'accepter sans arrière-pensée une langue à la fois indigène et étrangère ${ }^{2}$. L'image d'une langue peut entraîner des effets socioculturels plus larges sur l'identité des individus, des communautés et de la nation. Cette image naît de plusieurs éléments : le statut de ses locuteurs ou du groupe qui y est associé ; la capacité de la langue à être utilisée dans différents domaines sociaux, par exemple techniques, artistiques, familiaux ou publics; et la valeur symbolique ou pragmatique qu'elle comporte. Cette image est sujette aux changements, souvent mais pas toujours en tandem avec le déclin ou la persistance de la langue elle-même.

Évidemment, la langue en tant que notion se trouve souvent liée à celles des droits politiques et de l'identité nationale ou ethnique. Cependant, au sein de cette constellation puissante, la langue ne doit pas être regardée seulement comme une manifestation d'une ou des autres. Elle constitue une force en elle-même. Les locuteurs des langues minoritaires évoquent souvent l'idée que la langue soit ou ait une force spirituelle, te wairua o te reo [la force de vie de la langue] (Morgan, 2009) ou une âme (Ní Dhomhnaill, 1997 : 48). Colls soutient que :

[Languages] are living, breathing organisms holding the connections and associations that define a culture" [Les langues sont des organismes vivants qui contiennent les connexions et associations qui définissent une culture] (Colls, 2009).

Bien que les éventuels effets des médias ne soient toujours pas complétement analysés (Fishman, 2001 : 473 ; Cormack, 2007), il est clair qu'ils détiennent les qualités nécessaires pour interagir avec ces relations linguistiques.

Pour mieux comprendre l'image de la langue minoritaire auprès des locuteurs et des non locuteurs, il faut prendre en compte le pay-

2. "It is difficult not to be self-conscious about a language simultaneously native and foreign" (Deane, $1985: 13$ ). 
sage sociolinguistique et médiatique. Le concept de «l'image de la langue» comprend alors les éléments visuels projetés sur l'écran (domaine sociolinguistique, apparence des présentateurs, usage de dialectes différents) et aussi des éléments moins visibles (l'attitude du public, la relation psychologique avec une langue ancestrale nonparlée).

Cet article est basé sur mon travail de thèse, qui traite de l'utilisation de la langue minoritaire indigène sur l'écran et pendant le tournage. En étudiant le cas des chaînes de service public Māori Television $^{3}$ en Nouvelle-Zélande et TG4 en Irlande, j'ai examiné comment ces choix peuvent influencer l'image de la langue. J'ai également recherché comment de tels médias peuvent communiquer avec des non-locuteurs qui se considèrent comme appartenant à cette culture et cette langue, et qui veulent y être reconnectés ou refamiliarisés. Cette intervention voudrait décliner les enjeux de production télévisuelle en irlandais et en māori, dans le but de préparer le terrain pour une future exploration des médias audiovisuels contemporains en breton.

\section{La relation entre la langue et les structures de pouvoir/l'État}

N'importe quelle situation linguistique aujourd'hui trouve ses origines dans l'histoire du lieu. Si quelques personnes évoquent des arguments biologiques concernant la protection des langues (en parlant de 'diversité', de 'déclin' et de 'mort' (Crystal, 2002 : 32), il n'en est pas moins important de souligner que les langues peu répandues de nos jours le sont souvent suite à des décennies d'interventions politiques, militaires ou coloniales (May, 2009). Selon Kymlicka et Patton (2003), une politique de 'laissez-faire' (benign neglect) n'est autre qu'un refus de l'État-nation d'honorer une obligation éthique de réparer les dégâts du passé.

Depuis son indépendance politique, l'État irlandais considère l'irlandais comme première langue officielle, et garantit sa présence dans l'éducation nationale (Ireland, 1937). Cependant, seulement un

3. Māori Television est la chaîne de télévision nationale de la langue māori (www.maoritelevision.com). 
petit pourcentage de la population la parle couramment dans la vie quotidienne (Príomh-Oifig Staidrimh na hÉireann, 2011). En Nouvelle-Zélande, le māori est reconnu en tant que langue nationale depuis 1987, et la Couronne, la Nouvelle-Zélande étant membre du Commonwealth, est chargée de la protéger en tant qu'élément culturel (New Zealand, 1987). Ces deux pays accordent un statut spécifique à leurs langues minorisées ${ }^{4}$ par la colonisation anglaise, mais ceci ne suffit pas pour changer les habitudes linguistiques implantées chez les habitants depuis cette époque.

En Irlande, la langue fait partie de l'image de la nation (même si l'État n'a pas su la développer), tandis qu'en Nouvelle-Zélande, la langue (après une grande réticence) est catégorisée comme taonga (trésor), mais pas comme élément essentiel de l'identité nationale. Dès lors, la télédiffusion est confrontée à un dilemme de pertinence et se trouve déchirée entre deux approches : fournir un service communautaire pour des locuteurs, aider les apprenants et présenter le monde de la langue à un groupe national plus important qui ignore la culture de cette langue.

En Bretagne, la langue est soutenue en partie et dans des domaines particuliers par des mesures régionales, mais jusqu'à présent, elle ne bénéficie pas du soutien de l'État. En décembre 2004, le Conseil régional de Bretagne a reconnu le breton (et le gallo) «comme langues de la Bretagne historique» et depuis essaie de mettre en œuvre une politique favorable au multilinguisme (Rannvro Breizh, 2012). Cependant, faute de moyens, l'image du breton risque d'être plutôt folklorique que contemporaine, car elle reste limitée à certains domaines. Même les récentes tentatives pour créer une image de marque comme À l'Aise Breizh - qui ont bien réussi de façon publicitaire (Le Coadic, 1998) - conservent au personnage de bigoudène une conception traditionnelle et figée de ce qui constitue une identité bretonne. L'histoire de ces images dépend, comme celles des deux langues évoquées ci-dessus, des circonstances politiques, économiques et idéologiques.

4. J'utilise le terme 'minorisé', suivant Arana et al ("minoritised language", 2007), car ce terme est plus politisé que 'minoritaire' et évoque la causalité. 


\section{La relation entre la langue et ses (non-)locuteurs}

Après un long processus de dévalorisation, après une longue coupure générationnelle, le breton est en effet devenu une langue étrangère pour une majorité de Bretons. (Le Pipec, 2011)

Pour les bretonnants de naissance, la langue devrait être transparente, mais à cause de sa place dans le domaine public vis-à-vis du français, elle devient remarquable. Par remarquable, je veux dire que nul adulte, quel que soit son niveau de compétence, ne parle une langue minorisée dans un lieu public sans être conscient de ce statut de minorité. Pour les néo-bretonnants cela est encore plus marqué, dans la mesure où certains choisissent de parler en breton pour souligner leur identité de 'rebelle' ou leur culture - ils se servent de la langue pour communiquer non seulement leurs pensées, mais aussi une attitude ou une image.

Si une langue va être utilisée en tant que moyen normal ou non marqué de communication, il faut bien que son statut dans les domaines publics soit revendiqué. François Grin, spécialiste en économie des langues et vice-coordinateur du projet intégré EU DYLAN (Dynamique des langues et gestion de la diversité), constate que l'utilisation d'une langue dépend de trois facteurs : la compétence linguistique, un environnement convenable et la volonté de parler la langue (Grin, 2002). Si la transmission intergénérationelle et l'éducation peuvent augmenter les effectifs des locuteurs compétents, l'intervention de la communauté ou le soutien de l'État sont exigés pour créer des environnements où la langue puisse être parlée en public d'une manière non marquée. Peut-être le facteur le plus fuyant par rapport au soutien extérieur est cette troisième condition de Grin, la volonté de parler la langue, car celle-ci réside dans le cœur et les sentiments des locuteurs et des néo-locuteurs.

La relation à haute charge émotionnelle qu'ont les gens avec la langue minorisée demande une reconnaissance et de la sensibilité. McCloskey note que pour beaucoup, en Irlande, la langue fonctionne comme un 'paratonnerre symbolique' (2008: 78-9) ${ }^{5}$, représente

5. "symbolic lightning rod" (2008: 78-9). 
souffrance et confusion. Cela est souvent le cas dans les situations où un basculement linguistique forcé a eu lieu (Maui, 2007 ; Le Morvan, $2000:$ 133).

L'image des langues māori et irlandaise a été, pour les nonlocuteurs pendant le dernier siècle, souvent négative. Associée à un «manque de joie qui semblait être la condition sine qua non d'une culture 'authentique'» (Barry, 1994-5 : 34) ${ }^{6}$, une simplicité rurale et une ambiance étouffante de tradition moisie, la langue minorisée semblait n'avoir pas grand-chose à proposer à la personne venant de l'extérieur, et bien de jeunes locuteurs n'étaient pas convaincus de la place de leur héritage dans le monde contemporain.

Malgré l'indépendance politique et l'intervention de l'État en Irlande pour promouvoir la langue, elle n'a pas été 'normalisée' pour devenir partie intégrante de la vie quotidienne de la plupart des citoyens (McGreil \& Rhatigan, 2009). Les gens habitant en dehors des Gaeltacht $^{7}$ avaient tendance à voir l'irlandais comme quelque chose d' 'autre', soit la langue noble et poétique du passé, soit le code subversif d'activités paramilitaires. Dans les deux cas, il s'agit d'une langue symbole et non pas utilisable dans la vie de tous les jours.

Le breton, tout comme l'irlandais ou le māori, est pour une grande partie de la population, tiré entre deux images opposées : «un patois de ploucs qui n'a pas de valeur» et «l'expression nationale de l'âme bretonne» (Calvez, 2011). Ces deux images absolutistes ne perdureront pas si un environnement réaliste advient, où le breton est parlé pour des raisons ordinaires. Je considère l'espace virtuel des médias comme un bon point de départ pour créer un tel environnement.

\section{Quel est le rôle des médias audiovisuels vis-à-vis des langues minoritaires?}

Les choix et les possibilités permis par l'ère numérique ont été accueillis par les productions en langue minoritaire. Beaucoup de défis du passé (techniques, financiers) sont désormais moins pesants.

6. "dourness which seem[ed] to be the sine qua non of 'authentic' culture" (Barry, 1994-5: 34).

7. La Gaeltacht est une désignation officielle donnée à des endroits géographiques où l'irlandais est la langue quotidienne de la communauté. 
Mais la nouvelle ère de l'abondance (Ellis, 2000) peut poser d'autres problèmes. La prolifération des chaînes et la facilité d'acquérir des émissions internationales mènent à une situation où les médias en langue minoritaire risquent de se perdre dans un univers plus large que la concurrence nationale. Si la nouvelle technologie permet de rendre leurs émissions plus accessibles à un public plus large (avec des sous-titres, les rediffusions sur internet, la vidéo à la demande...), la baisse des effectifs des locuteurs sur le terrain (en tant qu'intervenants aussi bien que public) reste un enjeu réel.

Cependant, on voit que l'internet attire une activité hors proportion pour certaines langues minoritaires (Carlson, 2010). Indigenous Tweets, fondé par un Américain irlandophone tente de suivre le nombre d'interventions sur Blogspot et Twitter en langues indigènes de par le monde (Scannell, 2011) ${ }^{8}$. BrezhoweB est un bon exemple d'un service de diffusion en langue bretonne qui contourne les antennes pour atteindre un public sur internet. Disponible depuis quatre ans, cette chaîne est témoin de la capacité ingénieuse des producteurs en langue minorisée face aux défis de diffusion. Cet aspect exige beaucoup plus d'attention, mais pour des raisons d'espace, on y retournera dans un futur article.

Avant de passer à la télévision traditionnelle, constatons les progrès effectués par les radios. Les radios iwi (tribu) en Aotearoa, à l'instar des radios pirates et communautaires en France et en Europe, ont commencé à se développer pendant les années 1970 et 1980. Chaque pays étant sous un régime plus ou moins centralisé, l'État est intervenu pour régulariser l'utilisation des ondes nationales ${ }^{9}$ par des groupes de citoyens. Les autorités ont réagi différemment : la France a reconnu des radios associatives en limitant leur zone de diffusion et la Nouvelle-Zélande a gardé un système de radios $i w i$ locales pour respecter la variété des dialectes des $i w i$, et aussi pour des raisons techniques. En Irlande, la 'radio libre' du Conemara a laissé la place

8. Sur environ 140 langues, le Breton y figure en $18^{\mathrm{e}}$ position avec 82 'tweeters', et en $12^{\mathrm{e}}$ position dans la section blogs avec 25 (au 27 septembre 2012). (Pour le nombre des 'tweeters', l'irlandais est en $4^{\mathrm{e}}$ et te reo Māori en $24^{\mathrm{e}}$ position. Pour les blogs, l'irlandais est en $2^{\mathrm{e}}$ et te reo en $21^{\mathrm{e}}$.)

9. Cette conception de possession des ondes a été fortement contestée par des iwi Māori, qui pendant les années 1980 et 1990 ont mené un procès contre le gouvernement de la Nouvelle-Zélande jusqu'au Conseil privé en Angleterre. 
à une radio en irlandais d'envergure nationale, Raidió na Gaeltachta, en $1972^{10}$.

Dans tous ces cas, l'essentiel est de reconnaitre la relation entre les communautés de locuteurs et leurs médias locaux. Comme le soulignent Nettle et Romaine, il est peu efficace d'accorder un statut à une langue si ses locuteurs ne sont pas 'habilités' ('empowered') (Nettle et Romaine, $2000: 39-40$ ). Face à un paysage médiatique qui les ignore ou qui les traite de spécimens exotiques stéréotypés (Le Guénic, 2003), les locuteurs de la langue minorisée essayent de combler le manque de représentations avec leurs propres médias.

\section{Quel rôle pour la télévision par rapport à l'image de la langue?}

Si Anderson signale qu'il n'est parfois pas évident de distinguer un texte ou une image visuelle de l'acte politique de prendre parole ou de représenter un groupe (2006: 44) ${ }^{11}$, il faut aussi reconnaître le pouvoir des images télévisées. Car le télédiffuseur en langue minorisée effectue non seulement un changement de l'image de la langue pour des non-locuteurs, mais il a aussi le potentiel de changer la manière dont les locuteurs sont perçus.

La télévision, en tant que média domestique non menaçant, est facile d'accès et fournit aux non-locuteurs une nouvelle perspective sur une langue et une culture qui pourraient leur sembler une porte fermée. La télévision aide à montrer une image 'normale' de la langue, qui y joue un rôle non-politique (Cormack, 2000 : 396). Sa présence à la télévision a pour effet d'enlever une partie de la mystique (ou du radicalisme politique qui y est souvent associé) perçue par la majorité. Les locuteurs apprécient la diversité générique qui rend visible un élément de leur monde linguistique.

10. Ceci dit, le service, avec ces trois studios régionaux et destiné principalement aux habitants de la Gaeltacht, a souvent réussi à privilégier des perspectives locales et internationales, grâce à un réseau de locuteurs dans d'autres pays du monde.

11. "It is sometimes difficult to distinguish a text or a visual image from the political act of presuming to speak for, or represent, a group of people" (Anderson, 2006 : 44). 
La raison pour établir un service de télévision en irlandais (TG4) et en te reo māori (Mãori Television) n'était pas de mettre au monde un musée ou une archive, mais plutôt de donner vie à un média contemporain pour informer et divertir le public. Les médias constituent de puissants vecteurs pour l'image d'une langue, notamment dans des situations où, comme pour les chaînes citées ici, ils fournissent à beaucoup de citoyens leur seule opportunité de s'engager d'une manière continue avec la langue minorisée comme langue vivante. Pour un télédiffuseur qui fait «de la télévision en langue bretonne et non pas la langue bretonne à la télévision» comme disait Alain Bienvenu, responsable des émissions bretonnes à France 3 en 1989 (Robineau, 2001), la langue est présentée comme résolument ordinaire. Paradoxalement, cela aide à faire croître une reconnaissance de la communauté comme entité différente et diverse (Cormack, $2000: 393$ ).

Depuis les années 1960, beaucoup de groupes sociaux ont revendiqué la liberté de se représenter et de représenter les autres à travers leurs propres médias. Non seulement cette évolution constitue la reconnaissance et le respect de la langue, mais elle permet aussi une réelle croissance d'espoir pour l'avenir. En créant des emplois dans des domaines attirants, elle montre aux jeunes locuteurs que leur langue leur permettra non seulement de s'exprimer auprès de leur communauté, mais aussi de gagner leur vie. Même si en Bretagne, le nombre de jeunes locuteurs est moins important que les plus âgés (Broudic, 2009), leur proposer une voie professionnelle les encouragera à continuer à utiliser le breton pendant leur vie adulte.

\section{Crédibilité acoustique}

Si la représentation de la langue à l'écran ne reflète pas toujours l'usage contemporain (normalement on entendra plus la langue majoritaire hors tournage), elle aide à créer un nouvel univers potentiel pour les jeunes publics. La télévision confère à la langue une certaine «crédibilité acoustique» (Ó Tuathaigh, 2008), permettant aux enfants de considérer la langue comme quelque chose de contemporain à égalité avec d'autres productions culturelles (Howell, 1992 : 218). Thomas remarque aussi que quand il s'agit d'une population 
éparpillée, les médias de diffusion sont particulièrement importants pour que la communauté reste 'cohésive' (Thomas, 1978 : 90). Il faut bien prendre cette considération en compte car en Bretagne il est clair que les bretonnants de l'avenir seront plutôt une communauté virtuelle que territoriale (Broudic, 2009).

La question du territoire et des structures de pouvoir est très importante pour les médias en langue minorisée. En général, on considère qu'ils ont plus de chance d'atteindre leurs objectifs si les locaux de diffusion ou de décision sont proches des communautés des locuteurs. C'est le cas en Irlande où les studios de TG4 sont à l'ouest, dans une région Gaeltacht ${ }^{12}$, mais Māori Television se trouve à Auckland pour des raisons logistiques. Pour l'instant, une partie de la production des émissions télévisées en langue bretonne (les actualités) se fait à Brest, en Finistère, et une autre partie (principalement le divertissement) à Rennes, en Île-et-Vilaine. Le département du Finistère héberge une bonne proportion des bretonnants (Broudic, 2009). Mais les services ont beau être implantés près des locuteurs, les deux lieux subissent un contrôle central de Paris. Les décisions de rédaction et de placement des programmes ne s'effectuent pas toutes dans la région. Bien qu'éléments d'un service de télévision régionale, France 3 Iroise voit son identité régionale s'effondrer au profit des émissions générales nationales (Personnel de France 3, 2009). Il en va de même pour les créneaux horaires attribués aux émissions régionales (en français et en breton), qui sont souvent placées aux heures creuses de la grille ou, pour les émissions en breton, supprimées pendant certaines périodes de l'année ${ }^{13}$. Il est important que les médias soient proches de leurs publics, certes, mais on voit de cet exemple que la proximité géographique ne vaut pas une proximité linguistique ou idéologique. Si le siège du pouvoir est loin, les médias locaux manquent de pouvoir de décision concernant leur service vis-à-vis du public.

12. L'implantation dans un endroit ne correspond pas nécessairement à une acceptation du peuple (Fennell, 2001) et bien sûr, la situation géographique isolée de TG4 pose d'autres défis.

13. Notamment pendant les vacances scolaires (Personnel de France 3, 2009 ; Malesieux, 2012). 


\section{Glamour et grammaire}

Il existe deux arènes interdépendantes à prendre en compte quand on présente une langue minorisée à l'écran : le registre linguistique et l'image de la langue. Bien sûr, les mots «grammaire» et "glamour» ont la même racine. Le glamour des people de la télévision et la preuve que d'autres locuteurs existent au-delà de la salle de classe ou de la paroisse rurale rendent la langue plus attirante à des jeunes et à des néo-locuteurs. Quand on voit régulièrement à la télé toute une gamme de bons locuteurs dans différents contextes situationnels, l'image de leur langue est ancrée dans l'imaginaire des publics comme quelque chose de vivant et d'efficace dans la vie moderne - même pour ceux qui ne la parlent pas, ou qui n'ont pas la possibilité de croiser des locuteurs (Le Morvan, 2000 : 126).

Si la télévision en tant que media domestique se prête facilement à une communication informelle et conversationnelle, une langue minoritaire ne peut pas toujours se permettre ce luxe. Larry Parr de Māori Television fait référence à une sorte d'attitude «deux poids deux mesures» qui découle de la situation de pénurie pour les émissions en langue minoritaire, là où les émissions en anglais peuvent se servir d'une gamme large de styles et de registres. Cependant les programmes en langue māori sont censées être toujours grammaticalement correctes : "Cela retire la vie de la langue dans une certaine mesure» (Parr, 2007) ${ }^{14}$. L'exactitude linguistique ne suffit pas en soi pour inspirer le public et faire jaillir la vie. Il n'y a pas lieu de promouvoir une fausse image ou version de la langue, comme il peut se produire quand les présentateurs sont trop 'corrects' ou scénarisés.

Māori Television et TG4 présentent la langue minorisée dans des domaines inattendus et dans des cadres informels, ils mettent souvent en valeur de jeunes et beaux animateurs, ce qui modifie les vieux stéréotypes d'une langue moribonde. En même temps, il peut $\mathrm{y}$ avoir une tension entre les images traditionnelles et modernes des locuteurs des langues minoritaires (Hourigan, 2003 : 129), car le côté branché d'un animateur de télé peut en quelque sorte révéler un manque de respect pour l'intégrité des vraies sources de la langue. La (re)représentation de ces images et l'invention de nouvelles

14. "It's taking the life out of the language to some extent" (Parr, 2007). 
représentations des aspects du peuple et de la culture exigent une certaine délicatesse.

\section{Découverte et refamiliarisation}

Les locuteurs peuvent se sentir 'chez eux', en regardant un service médiatique varié. Pour ceux qui apprennent la langue, la télévision peut initier un voyage de reconnexion et de refamiliarisation.

Une telle approche trouve son écho dans le stratagème connu de Barry Barclay 'talking in/out' (s'adresser à nous/ à eux) (Barclay, 1990 : 74). Selon Barclay, il existe deux façons de mettre une culture minorisée à l'écran : une version locale qui parle à la communauté selon leurs propres conditions ('talking out'/ 's'adresser à nous'), ou un modèle plus orienté vers le tourisme ou l'éducation qui dit l'évidence pour attirer l'intérêt des gens extérieurs à la culture ('talking out'/ 's'adresser à eux').

L'étude faite par Arapera Ngaha montre que l'importance attribuée à la langue et à la culture en tant que marqueurs d'identité peut se transmuer (Ngaha, 2005) et c'est dans cette optique-ci que je prends le terme 'refamiliarisation', car il n'insiste pas sur l'usage et la compétence linguistique, mais plutôt sur la qualité de la relation entre la personne et la langue. Il est évident que cette relation ira plus loin si la personne sait parler, mais cela n'est pas une condition préalable.

Si les gens doivent utiliser une langue minorisée en public sans honte, il faut qu'ils la voient comme un moyen 'normal' de communication, et la nature domestique et omniprésente de la télévision peut contribuer à une 'normalisation d'image' en fournissant une gamme de genres, y compris des émissions 'banales' ou de divertissement ${ }^{15}$. Bien que moins efficace que la transmission intergénérationnelle, l'usage de la langue minorisée dans les médias aide à créer un environnement où une telle transmission peut avoir lieu. En tant que «promoteur de grand prestige de la culture orale» (Fiontar, $2009: 29)^{16}$, la télévision a le potentiel de sensibiliser les non-locuteurs, tout en renforçant l'estime de soi des locuteurs.

15. Cormack note qu'une présence médiatique contribue à 'légitimer' une langue aux yeux du public (Cormack, 2007 : 53-5).

16. "high prestige promoter of oral culture" (Fiontar, $2009: 29$ ) 
L'effet pratique des médias en langues minoritaires (MLM) est la refamiliarisation d'une langue. Si les termes 'renouvellement', 'revitalisation' ou 'récupération' sont bien utiles dans des situations où la langue est en grave danger quand de nombreuses personnes n'ont plus de lien avec elle, 'refamiliarisation' décrit le processus où une langue à moitié oubliée est rendue à nouveau familière. Pour la refamiliarisation, l'essentiel est de reconstruire la relation que les gens ont avec la langue. À la différence de la normalisation, qui implique une large acceptation et l'usage sociétal d'un standard, la refamiliarisation parle des gens qui (re)prennent connaissance avec leur propre variété, afin qu'ils se sentent en mesure de l'utiliser sans trop d'arrière-pensées. On utilise différents dialectes, on représente et on inclut, on évite trop de néologismes académiques (même s'il faut prendre des mots francisés, qui, grâce à leur usage à long terme, semblent moins formels que les nouvelles expressions issues des livres), et il est préférable de parler couramment avec quelques fautes que de prononcer les mots un par un avec une grammaire impeccable.

\section{Quel rôle pour la télévision par rapport à l'utilisation de la langue?}

Mike Cormack souligne que l'on peut nourrir de grands espoirs irréalistes par rapport au potentiel de la télévision pour la restauration de la langue, car il est évident que les raisons pour l'utilisation de la langue dans la vie sociale sont diverses, différentes et complexes (Cormack, 2007 : 62). Beaucoup dépendent de la façon dont le public interagit avec le diffuseur. Comme le dit Cormack, «l'important n'est pas le contenu des medias en tant qu'une sorte de force isolée, ni les institutions et organisations médiatiques comme pouvoirs hégémoniques, mais plutôt l'espace entre les médias et leurs usagers 》 (Cormack, $2007: 65)^{17}$.

Cet espace est un lieu où la langue reprend vie. Bien qu'on ne puisse pas facilement prouver les effets des medias sur la qualité et la quantité des locuteurs, il semble que les téléspectateurs 'attrappent' et utilisent des expressions entendues souvent lors des

17. "The emphasis here is not on media content as some kind of free-standing force, nor on media institutions and organisations as hegemonic powers, but rather on the space between the media and their users" (Cormack, $2007: 65$ ). 
émissions (Kelly-Holmes, 2001 : 4 ; Lysaght, 2004 : 151 ; Mane, 2009). On voit l'influence des médias en langue majoritaire, ou 'la privation d'avenir' des cultures minoritaires (Lafont, 1982 : 18). Pádraig Ó Mianáin élève ses enfants irlandophones dans un lieu principalement anglophone, et considère TG4 comme un intrant langagier supplémentaire pour leur montrer qu'un monde autre que le foyer familial existe en cette langue :

Tá ról le himirt ag an teilifís Ghaeilge i múnlú agus i bhforbairt theanga na bpáistí, ag cur an tsaoil ina láthair agus ag tabhairt friotail dóibh leis an saol sin a láimhseáil" [La télévision en irlandais joue un rôle à former et développer la langue des enfants, à leur présenter le monde et à leur fournir des phrases avec lesquelles ils peuvent traiter ce monde-là] (Ó Mianáin, $2008: 45)$

Ó Mianáin considère que la langue informelle de la télévision est plus utile dans la vie quotidienne que l'irlandais d'école que les enfants apprennent plus tard. Il existe aussi des témoignages anecdotiques concernant les effets de la télévision sur des jeunes locuteurs de te reo Mãori en Nouvelle-Zélande, où les écolières s'envoient des textos en utilisant les nouveaux mots qu'elles ont appris de Māori Television (Edwards, 2009) ${ }^{18}$.

Une question très importante pour l'utilisation de la langue dans les médias est bien sa pratique sur le lieu de travail. On ne peut discuter de cette question en raison de contraintes d'espace. Qu'il suffise de dire qu'il existe quelques maisons de production qui ont pris la décision d'utiliser leur langue (qu'elle soit l'irlandais, te reo ou le breton) comme langue de travail ${ }^{19}$. Il faut constater qu'en général, comme dans le reste des domaines actuels, la langue minorisée est parlée seulement si la volonté y est, et tout dépend de l'attitude (et de la compétence !) des professionnels. Comme le remarque Joshua Fishman, toute tentative «d'inverser le basculement linguistique

18. Cela correspond avec les études effectuées par O'Connell sur le rôle de la télévision pour renforcer un nouveau vocabulaire (O'Connell, $2003: 60$ ).

19. Notamment Nemeton TV (Irlande), Kura Productions (Nouvelle-Zélande) et Kalanna (Bretagne). 
implique un renouvellement de la réalité sociale et c'est très difficile pour les minorités d'y arriver» (Fishman, $1991: 411)^{20}$.

\section{Conclusion}

Pour renouveler la réalité sociale, il faut d'abord une vision. Bien des promoteurs des langues minorisées n'ont rien contre un bilinguisme plus paritaire. Disons qu'avec deux yeux, on peut voir plus clair. Si la vision est celle d'une société où deux langues peuvent exister dans le domaine public, il faudra bien que la langue minorisée soit plus présente dans les médias. Une façon de modifier la réalité sociale est de renouveler les images que l'on projette et avec lesquelles la société interagit pour se créer des identités. Les médias constituent des outils puissants pour un tel travail, car ils ont la possibilité d'atteindre plusieurs publics. La télévision est facile d'accès, à la fois pour des raisons techniques (ubiquité) et linguistiques (la présence des images et des sous-titres rendant les émissions plus abordables à des non-locuteurs que des émissions de radio, par exemple). Pour cela, il faut bien sûr une volonté politique de soutenir la diffusion variée et consistante. On pourra s'appuyer sur les arguments de l'obligation éthique d'un État de réparer les dégâts linguistiques occasionnés par ses actions dans le passé ; ou bien de la tradition de la radiodiffusion de service public ou de l'idéal des services régionaux, même si ces trois approches semblent avoir peu d'emprise sur bien des gouvernements contemporains.

Il est clair qu'il existe actuellement en Bretagne plusieurs publics qui seraient intéressés par un tel projet : les locuteurs natifs âgés qui ont droit à un service médiatique plus complet dans leur langue, les gens qui apprennent le breton pour des raisons identitaires ou culturelles, qui ont besoin d'entendre et de voir la langue pour pouvoir progresser, et finalement les jeunes et les enfants qui doivent pouvoir vivre leur breton en dehors de la salle de classe. Ce dernier groupe sera celui qui portera la langue dans l'avenir et qui en fera une réalité vivante.

20. "Reversing Language Shift... implies remaking social reality and that is very hard for minorities to do" (Fishman, $1991: 411$ ). 
Si les années 1980 et 1990 ont vu des tendances décentralisatrices s'imposer partout en Europe et ailleurs, la France garde toujours une politique centralisée au début du $\mathrm{XXI}^{\mathrm{e}}$ siècle. Il faut souligner que la relation entre la langue française et les Français est bien différente de celle qui existe entre la langue anglaise et les peuples du monde anglophone. Le français est souvent considéré d'une manière idéologique comme vecteur des valeurs de la République, comprenant identité nationale et citoyenneté ${ }^{21}$. La plus grande différence est sans doute ce qui en découle : les médias dépendent d'un pouvoir centralisé en langue française, tandis que dans les deux autres pays mentionnés dans cet article, le gouvernement national (pour des raisons diverses) a cédé une partie de son autorité sur le contenu des médias aux locuteurs de la langue minorisée. On voit alors qu'en Bretagne, les chaînes et les producteurs d'émissions en langue bretonne sont confrontés à des défis similaires à leurs homologues d'Irlande et de Nouvelle-Zélande, mais qu'il leur manque l'autonomie et les fonds pour y faire face de façon concrète et soutenue.

\section{Bibliographie}

ANDERSON Benedict, Imagined communities : reflections on the origin and spread of nationalism, London, New York, Verso, 2006.

Arana Edorta, Patxi AzPILlaga, Beatriz Narbaiza, «Linguistic Normalisation and Local Television in the Basque Country», in Mike CORMACK \& Niamh Hourigan (ed.), Minority Language Media: Concepts, Critiques, and Case Studies, Clevedon, Buffalo, Multilingual Matters, 2007, p. 151-167.

BARCLAY Barry, Our Own Image, Auckland N.Z., Longman Paul, 1990. BARRY Shane, Film Ireland, Jan. 1994, p. 34.

«BrezhoweB - Bienvenue sur Brezhoweb, la première chaîne TV 100 \% en langue bretonne !», consulté le 27/09/2012.

BROUDIC Fañch, et TMO régions (Firm), Parler breton au XXIe siècle : le nouveau sondage de TMO régions, Brest, Emgleo Breiz, 2009.

21. Ironiquement, ces charges idéologiques ont été prises par la langue indigène nationale en Irlande et en Nouvelle-Zélande. 
BUTLER Eoin, «Interview with Rónán Mac Aodha Bhuí: 'MC Muppet is a rapper from Connemara whose lyrics are very sexual in content. The Irish language is a very poetic language'», The Irish Times, 13 Mar. 2010, consulté le 07/07/2010.

CALveZ Ronan, «De quoi breton est-il le nom?», dans Langues de France, langues en danger : aménagement et rôle des linguistes, Laboratoire Dynamique Du Langage (DDL, UMR 5596) Laboratoire Interactions, Corpus, Apprentissages, Représentations (ICAR, UMR 5191) - Institut Pierre Gardette. Institut des Sciences de l'Homme (ISH), Université de Lyon, L'Harmattan, 2011. Délégation générale à la langue française et aux langues de France (DGLFLF).

CARLSON Betty, «Internet and minority languages | Language Translation», consulté le 25/09/2012.

LE COADIC Ronan, L'identité bretonne, Rennes (France), Terre de Brume Éditions / Presses universitaires de Rennes, 1998.

CoLLS Tom, «The death of language?», BBC Today 2009, consulté le $15 / 12 / 2009$.

CORMACK Michael, «Introduction: Studying Minority Language Media», in Mike CORMACK \& Niamh Hourigan (ed.), Minority Language Media: Concepts, Critiques, and Case Studies, Clevedon, Buffalo, Multilingual Matters, 2007, p. 1-16.

CORMACK Mike, «Minority Language Media in a Global Age», Mercator Media Forum 4 (2000), p. 3-15.

CORMACK Mike, "The Media and Language Maintenance», in Mike CORMACK \& Niamh HOURIGAN (ed.), Minority Language Media: Concepts, Critiques, and Case Studies, Clevedon, Buffalo, Multilingual Matters, 2007, p. 52-68.

CRYSTAL David, English as a Global Language, Cambridge University Press, 2003.

DEANE Seamus, Celtic revivals: essays in modern Irish literature, 18801980, London, Boston, Faber and Faber, 1985.

EDWARDS Hone, «Presentation», Māori Television Rautaki Reo hui/ language strategy meeting, Auckland, 2009.

ELLIS John, Seeing things: television in the age of uncertainty, London [etc.], I.B, Tauris, 2000.

FENNELl Cilian, former Head of Programmes, TG4 - Entretien du 24/06/2001. 
FIONTAR, Dublin City University. 20-Year Strategy for the Irish Language. Prepared for the Department of Community, Rural and Gaeltacht Affairs, 2009, consulté le 13/07/2010.

FISHMAN Joshua, Can threatened languages be saved?: reversing language shift, revisited: a 21st century perspective, Clevedon, Multilingual Matters, 2001.

FISHMAN Joshua, Reversing Language Shift: theoretical and empirical foundations of assistance to threatened languages, Clevedon, Philadelphia, Multilingual Matters, 1991.

GRIN François et al., Support for Minority Languages in Europe. Report to the European Commission, European Commission, 2002, consulté le 22/08/2010.

LE GUÉNIC Michel, Nos régions selon Jean-Pierre Pernaut : pétainisme ou pittoresque?, Nice, France Europe Éditions Livres, 2003.

HoURIGAN Niamh, Escaping the Global Village: media, language \& protest, Lanham Md., Lexington Books, 2003.

HowelL W., «Minority-Language Broadcasting and the Continuation of Celtic Culture in Wales and Ireland», in Stephen RigGINS (ed.), Ethnic Minority Media, an International Perspective, 1992, p. $217-$ 242.

IRELAND, Bunreacht na hÉireann / Constitution of Ireland, 1937.

KĀRETU Tīmoti, "Language and protocol of the Marae», in Michael KING (ed.), Te Ao Hurihuri: Aspects of Māoritanga, Auckland, Reed Books, 1992, p. 29-42.

Kelly-Holmes Helen, Minority Language Broadcasting: Breton and Irish, Clevedon, Multilingual Matters, 2001.

KYMLICKA Will, et Alan PATTEN, Language rights and political theory, Oxford, New York, Oxford University Press, 2003.

LAFONT Robert, «La privation d'avenir ou le crime contre les cultures», dans Ernest BERTHET et al. (éd.), Langue dominante, langues dominées, Paris, Edilig, 1982, p. 15-36.

LYSAGHT Ruth, «Pobal Sobail: Ros na Rún, TG4 and Reality », in Ruth BARTON \& Harvey O'BRIEN (ed.), Keeping it Real: Irish film and television, London, New York, Wallflower Press, 2004, p. 147-158.

LYSAGHT Ruth, «Teanga \& Tikanga: A Comparative Study of National Broadcasting in a Minority Language on Māori Television and Teilifís na Gaeilge», 2010.

MAC GRÉIL Mícheál, Fergal RHATIGAn, The Irish Language and the Irish People: report on the attitudes towards, competence in and use of the Irish language in the Republic of Ireland in 2007-'08, 
Maynooth Ireland, Survey and Research Unit, Department of Sociology, National University of Ireland Maynooth, 2009.

MANE Jo, «Pāho Māori: the impact of Māori language broadcasting on Māori language survival», 2009.

MAUI Erana, Personal communication, 2007.

MAY Stephen, Language and minority rights: ethnicity, nationalism and the politics of language, London, Routledge, 2008.

McCloskeY James, "Irish as a World Language», in Brian Ó CONCHUBHAIR (ed.), Why Irish?: Irish Language and Literature in Academia, Galway, Arlen House, 2008.

MoRgAn Eruera, Te Arawa, Tainui - Tumuaki Whakaaturanga / Head of Programming for Te Reo channel - Entretien du 6/10/2009.

LE MORVAN Murielle, «Les informations télévisées en langue bretonne : réflexions et expérience d'une journaliste bilingue», Mercator Media Forum 4 (2000), p. 125-137.

Nettle Daniel \& Suzanne Romaine, Vanishing Voices: the extinction of the world's languages, New York, Oxford University Press, 2000.

NeW ZEALAND, Māori Language Act, 1987.

NGAHA Arapera, «Language and Identity in the Māori Community: Without the reo who am I?», in Paul KERSWILL (ed.), Researching Language Use and Language Users, Linguistic Society of New Zealand, 2005, p. 27-48.

Ní DHOMHNAILl Nuala, «Why I choose to write in Irish, the corpse that sits up and talks back», in Susan SHAW SAILER (ed.), Representing Ireland Gender, Class, Nationality, Gainesville, University Press of Florida, 1997, p. 45-56.

Ó LAOIRE Lillis, On a rock in the middle of the ocean: songs and singers in Tory Island, Ireland, Lanham Md., Scarecrow Press, 2005.

Ó MiAnÁIN Pádraig, «An 'Mheán' - Ghaeilge: Caighdeáin Teanga i gCláir Óige TG4», in Eithne O'CONNELL, John WALSH, \& Gearóid DENVIR (ed.),TG4@10: deich mbliana de TG4/ten years of TG4, Indreabhán, Cló Iar-Chonnachta, 2008, p. 42-50.

Ó Tuathaigh Gearóid, "The State and the Irish Language», in Caoilfhionn Nic PHÁIdín \& Seán Ó CEARNAigh (ed.), A New View of the Irish Language, Baile Átha Cliath, Cois Life, 2008, p. 26-42. O'CONNELL Eithne, Minority language dubbing for children: screen translation from German to Irish, Frankfurt, Peter Lang, 2003.

PARR Larry, Ngāti Raukawa, Muaupoko - former General Manager Programming at Māori Television - Entretien du 13/12/2007. 
LE PIPEC Erwan, «Le breton, langue-totem ?», UBO, 2012, consulté le 27/09/2012.

Príomh-Oifig Staidrimh na hÉireann / Central Statistics Office, Ireland. «Census - CSO - Central Statistics Office Ireland», 2011, consulté le 10/08/2012.

RANNVRO BREIZH, «> Langues de Bretagne - Région Bretagne», consulté le 27/09/2012.

ROBINEAU Soazig, «Entretien avec Alain Bienvenu, ancien responsable des émissions bretonnes sur France 3», janv. 2001.

SCANNELL Kevin, «Indigenous Tweets», consulté le 27/09/2012.

SinHA Shalini, «Love our Language», The Irish Times 22 Nov. 2005.

THOMAS Ned, The Welsh Extremist, Aberystwyth, Y Lolfa, 1978.

WATERS John, «New Irish may save language», The Irish Times 6 June 2006. 